Annuaire suisse de politique de développement

25-2| 2006

Paix et sécurité : les défis lancés à la coopération internationale

\title{
De l'opérationnel au plaidoyer. Contributions des organisations suisses de développement à la promotion de la paix
}

\section{Maya Krell}

\section{(2) OpenEdition}

\section{Journals}

Édition électronique

URL : http://journals.openedition.org/aspd/267

DOI : 10.4000/aspd.267

ISSN : 1663-9669

Éditeur

Institut de hautes études internationales et du développement

Édition imprimée

Date de publication : 1 octobre 2006

Pagination : 159-175

ISBN : 2-88247-064-9

ISSN : 1660-5934

\section{Référence électronique}

Maya Krell, « De l'opérationnel au plaidoyer. Contributions des organisations suisses de

développement à la promotion de la paix », Annuaire suisse de politique de développement [En ligne], 25-2 | 2006, mis en ligne le 25 janvier 2010, consulté le 07 septembre 2020. URL : http:// journals.openedition.org/aspd/267; DOI : https://doi.org/10.4000/aspd.267 


\section{De l'opérationnel au plaidoyer}

Contributions

des organisations suisses de développement à la promotion de la paix

Maya Krell*

\section{Introduction}

«L'ennemi n'est plus au gouvernement.» C'est par cette phrase que la présidente de l'organisation argentine des Mères de la place de Mai, Hebe de Bonafini, a annoncé en janvier 2006 la $25^{\text {e }}$ et dernière marche de 24 heures. "Le président a ouvert les portes, il a fait ce que personne n'attendait plus. C'est pourquoi nous arrêtons cette marche de protestation annuelle. ${ }^{1}$

Il y a trente ans, les militaires d'Argentine accédaient au pouvoir par un putsch. Ils s'y sont maintenus jusqu'en 1982. Pendant cette période, quelque 30'000 personnes ont disparu dans le pays. En avril 1977, les mères de disparus (les madres) se rencontraient pour la première fois et organisaient leur marche annuelle alors que régnait encore la dictature militaire. Après la dictature, les madres tentèrent en vain de s'entretenir avec le gouvernement. En 1986, le président Raúl Alfonsín décréta l'amnistie et sous son successeur, Carlos Menem, la maison des madres fut cambriolée à plusieurs reprises pour y récupérer les clichés incriminant les militaires. Ce n'est qu'en 1993, dix ans après la fin de la dictature militaire, que les conditions changèrent. Après l'élection de Néstor Kirchner à la présidence en 2003, la loi d'amnistie fut annulée et le président s'excusa publiquement pour la terreur pratiquée par l'Etat.

$\mathrm{Au}$ cours de leur combat harassant pour obtenir des informations sur le sort de leurs enfants disparus, les madres ont reçu le soutien d'organisations étrangères, entre autres de la Suisse. Dans les années 1980 et 1990, leur combat pour la justice leur valut plusieurs prix, dont le prix de l'UNESCO pour la formation à la paix (1999). Si les marches annuelles de 24 heures appartiennent maintenant au passé, les mères continueront malgré leur grand âge (les pionnières sont aujourd'hui octogénaires) de manifester et de rappeler l'injustice et la violence d'alors et d'exiger que les responsables soient amenés à rendre des comptes.

L'histoire des Mères de la place de Mai illustre de façon impressionnante la forme que peut prendre l'engagement pour une société non violente et quel rôle les groupes et organisations de la société civile locale ou étrangère peuvent y jouer. L'Argentine n'a pas encore résolu tous ses problèmes et le travail de mémoire sur son passé brutal n'est pas achevé, mais cette histoire démontre que des années d'efforts peuvent aboutir à des résultats longtemps inimaginables.

Entraide protestante suisse (EPER), Bureau des activités en faveur de la paix, Zurich.

J. Vogt, «Die Erinnerung verblasst nie», WochenZeitung, 2. Februar 2006, p. 11 (ici et tout au long de ce chapitre, les citations extraites de documents non francophones ont été traduites par nos soins N.D.E.). 
Elle montre également que le processus peut prendre beaucoup de temps et qu'il dépend de personnes et d'institutions qui, malgré les revers et les désillusions, croient le changement possible et y œuvrent. Cette histoire soulève aussi une question: Comment se fait-il que le processus ait été si lent? Pourquoi le prix du succès est-il si élevé? N'y aurait-il pas de méthodes plus efficaces pour triompher de la violence et de l'arbitraire?

Cette histoire montre par ailleurs qu'il y a différentes manières d'aborder les questions de sécurité et de résolution politique - plutôt que militaire - des conflits. Ainsi, aujourd'hui, le terme «promotion de la paix» n'évoque plus vraiment la lutte contre les dictatures; il nous fait plutôt réfléchir à la manière de mettre fin à la violence, aux conflits nationaux, aux guerres pour le contrôle de territoires et de ressources, aux attaques terroristes et aux interventions militaires, comme par exemple en Irak.

Notre texte récapitule les mesures de promotion de la paix des œuvres suisses d'entraide $^{2}$ et en montre les processus d'apprentissage, les champs de tension et les défis. Les principes et mesures de promotion de la paix peuvent se classer en quatre grandes catégories:

$\checkmark$ les programmes de promotion de la paix de plusieurs œuvres suisses d'entraide dits de parrainage, le plus souvent cofinancés par le Département fédéral des affaires étrangères (DFAE);

$\checkmark$ les programmes et projets de promotion de la paix de quelques organisations, ayant explicitement pour objectifs la promotion de la paix ou la gestion des conflits et conduits en partenariat avec les organisations locales de différents pays du Sud et de l'Est;

- les programmes de développement comprenant des éléments ciblés de promotion de la paix tels que des offres intégrées de médiation ou de formation continue sur la gestion des conflits, de prévention de la violence, mais aussi de défense des droits humains;

- le travail de plaidoyer (advocacy) et le lobbying pour la politique et la promotion de la paix.

Les activités tenant compte des conflits (conflict sensitive) et l'approche fondée sur les droits humains (human rights-based approach) jouent un rôle essentiel dans le programme d'action de la majorité des œuvres d'entraide. Comme ces approches sont étroitement liées à la promotion de la paix, elles seront traitées plus loin dans deux sections indépendantes.

Un regard plus aiguisé

sur la dynamique des conflits et les processus de paix

Conformément à leur mandat, leur tradition et leurs convictions, les œuvres suisses d'entraide placent l'accent sur l'aide d'urgence et les programmes de développement. Les organisations suisses de développement réunies dans l'organisation Alliance Sud (Communauté de travail Swissaid, Action de Carême,

2 Ce texte fait état de l'expérience des œuvres d'entraide de Suisse rattachées ou représentées à l'organisation Alliance Sud ou au Centre pour la promotion de la paix (KOFF). 
Pain pour le prochain, Helvetas, Caritas, EPER) soulignent l'interdépendance du développement et de la paix ${ }^{3}$. Les Eglises défendent «la justice, la paix et la préservation de la création», vision que partagent les œuvres d'entraide confessionnelles suisses et qui va dans le même sens.

Les investissements dans le développement, l'engagement pour l'équité des rapports entre les pays industrialisés et les pays en développement ainsi que l'habilitation (empowerment) de groupes sociaux défavorisés ne débouchent toutefois pas automatiquement sur des rapports pacifiques et non violents. Si certaines organisations de développement, en général issues des milieux ecclésiastiques, soutiennent depuis longtemps des projets de prévention de la violence et des projets de paix dans différents continents, ce n'est que depuis la fin de la guerre froide, le génocide du Rwanda, l'escalade des conflits armés au cours des années 1990 et les missions de paix difficiles et souvent insuffisantes des Nations unies que les organisations de développement ont étudié de plus près la dynamique des conflits et la dynamique de la paix dans lesquelles elles évoluent. Certaines œuvres d'entraide telles que Caritas, le Christlicher Friedensdienst (CFD), l'Entraide protestante suisse, Helvetas, Action de Carême et la Croix-Rouge suisse ont ouvert ces dernières années des bureaux de promotion de la paix et de transformation des conflits afin de renforcer leur stratégie, le conseil et la formation dans ces domaines.

De nombreuses publications et études sur la paix et les conflits, le financement étatique de projets de promotion de la paix et la création de nouvelles organisations de travail opérationnel pour la paix ont également permis de mieux délimiter les perspectives envisageables. En particulier, le débat interne et la stratégie des œuvres d'entraide ont beaucoup profité de Do No $\mathrm{Harm}^{4}$, de l'étude de Mary Anderson et Lara Olson sur l'efficacité des mesures en faveur de la paix ${ }^{5}$, ainsi que des travaux de Jean-Paul Lederach ${ }^{6}$ (voir la contribution de Thania Paffenholz au présent ouvrage).

\section{Premiers programmes communs pour la paix}

La revalorisation de la promotion civile de la paix, y compris par les autorités fédérales - par exemple par la constitution d'un pool d'experts, le renforcement de la Direction politique et de la Direction du développement et de la coopération (DDC) au Département fédéral des affaires étrangères (DFAE) avec leurs services spécialisés, la création du Centre pour la promotion de la paix (KOFF) comme plate-forme des ONG et des bureaux fédéraux ainsi que par la nouvelle

3 P. Hug, sur mandat de la Communauté de travail Swissaid, Action de Carême, Pain pour le prochain, Helvetas et Caritas, Entwicklungspolitik ist die Friedenspolitik des 21. Jahrhunderts, 2000. Caritas, Alliance pour la paix, 2000.

4 La fin des années 1990 a vu le rapprochement de la science et de la pratique grâce en partie au livre de M.B. Anderson Do No Harm: How Aid Can Support Peace - or War, Boulder, Co, Lynne Rienner, 1999. Il s'agit d'une analyse du travail de développement conduit par Mary Anderson et son bureau de conseil Collaborative for Development Action ainsi que des effets directs et indirects des transferts de ressources en situation de crise.

5 M.B. Anderson, L. Olson, Confronting War: Critical Lessons for Peace Practioners, Cambridge, Mass., The Collaborative for Development Action, 2003.

6 J.P. Lederach, Building Peace: Sustainable Reconciliation in Divided Societies, Washington, DC, United States Institute of Peace Press, 1997. 
base juridique de $2003^{7}$ - ont permis de renforcer le dialogue entre les œuvres d'entraide et les services fédéraux.

Les premières collaborations multistakeholder de la promotion suisse de la paix - c'est-à-dire les premiers programmes communs à plusieurs œuvres d'entraide et services du Département des affaires étrangères - sont toutefois plus anciennes. L'initiative en est venue des deux côtés. Lors des négociations de paix au Chiapas, au début des années 1990, ce sont tout d'abord les œuvres d'entraide des Eglises qui ont demandé une aide à l'Etat fédéral suisse. Dans le programme de paix pour la Colombie, ce sont les services de la Confédération qui ont demandé aux ONG de renforcer l'engagement de la société civile pour parvenir à une solution politique au conflit armé qui durait depuis des décennies. C'est ainsi que diverses organisations de développement, un institut universitaire et des organisations des droits humains ont collaboré dans le cadre du programme SUIPPCOL ${ }^{8}$, largement financé par le DFAE.

Sur la base de ces premières expériences positives de conduite de programmes communs, et sachant que la promotion de la paix ne pouvait être pilotée avec succès que sous forme d'alliances, de nouveaux «programmes de parrainage d'ONG» ont suivi, comme, par exemple, la participation d'œuvres d'entraide protestantes suisses à une mission d'observation de la société civile en Palestine et en Israël. La difficile application des accords de paix au Guatemala et l'aggravation de la situation au Zimbabwe ont également incité plusieurs œuvres d'entraide à nouer des alliances œcuméniques.

\section{Promotion bilatérale de la paix et du développement}

Parallèlement à ces programmes de paix communs, les ONG suisses ont aussi soutenu des projets bilatéraux dans plusieurs régions frappées par les conflits ou la violence: soutien à des groupes de défense des droits humains, forums de discussion, cours et formation à l'approche constructive des conflits, missions d'observation, renforcement des ONG dans leur participation aux processus de transformation et de démocratisation. Dans ces projets, les organisations encouragent également la bonne gouvernance, le respect de l'Etat de droit et le renforcement de la négociation non violente dans les conflits d'intérêts.

Concrètement, les œuvres d'entraide encouragent, par le truchement de leurs organisations partenaires, une culture constructive des conflits et de la paix, basée sur la coopération (p. ex. par la formation à la paix). Cet engagement permet d'intervenir dans certains conflits, par exemple en cherchant les solutions acceptables par toutes les parties ou en servant de médiateur. Cela est possible lorsque les organisations partenaires ont accès aux différentes parties et sont réputées intègres.

En ce qui concerne l'aide d'urgence en cas de guerre ou de crise aiguë, les œuvres d'entraide contribuent à la protection immédiate des victimes de la violence. Elles secondent les forces de paix de la région et aident la société civile à

7 Loi fédérale sur des mesures de promotion civile de la paix et de renforcement des droits de l'homme et leurs crédits-cadres, en vigueur depuis le $1^{\text {er }}$ mai 2004.

8 Programa Suizo para la Promoción de la Paz en Colombia (SuIPPCOL), Friedensförderung der Schweiz in Kolumbien. Programm zur Stärkung der Zivilgesellschaft, 2005-2007 (version brève), Luzern, 2004. 
participer au processus de paix à court, moyen et long terme. Elles soutiennent aussi les organisations qui veillent au respect des principes de la paix durable (p. ex. le droit humanitaire international, les droits humains) et interviennent auprès du gouvernement suisse pour qu'il suive une politique de paix cohérente.

\section{Promotion de la paix comme élément complémentaire}

La promotion de la paix et la transformation des conflits, le fait donc de «travailler sur les conflits» (voir la contribution de Thania Paffenholz au présent ouvrage) est perçu aujourd'hui par la majorité des œuvres suisses d'entraide comme un complément aux activités traditionnelles de coopération au développement et, dans l'idéal, au développement rural, à l'exploitation durable des ressources, à la formation, aux rapports entre la société civile et l'Etat, à la sécurité alimentaire, à la sécurité du revenu, mais est également lié à l'aide d'urgence, compte tenu du fait que, selon le mandat et les compétences centrales, les priorités seront différentes.

Il est important pour la promotion de la paix de disposer d'une bonne vue d'ensemble, de choisir une bonne orientation stratégique comme des mesures et projets adaptés aux pays preneurs, et de bien les articuler. Le dénominateur commun des œuvres d'entraide, c'est la notion de «paix positive»: «La paix durable n'est pas que l'absence de violences personnelles et directes; elle implique également l'absence de violences structurelles, indirectes; elle est synonyme de justice sociale, de sécurité humaine et de stabilité structurelle. On parle de paix lorsque tous les membres d'une société peuvent satisfaire leurs besoins fondamentaux matériels et culturels. ${ }^{9}$ Cette définition repose sur la conviction et sur l'expérience que les mesures de promotion de la paix ne suffisent pas à elles seules à garantir une paix durable; il s'agit également de soutenir le changement social, politique et économique à différents niveaux.

En tant qu'organisations non gouvernementales, les œuvres suisses d'entraide se comprennent avant tout comme partenaires de la société civile du Sud et de l'Est. Elles se concentrent - souvent indirectement - sur l'habilitation (c'est-àdire le soutien à la participation de tous les secteurs de la population), la justice sociale, l'établissement de liens économiques, une culture constructive du conflit et le renforcement de l'Etat de droit ${ }^{10}$.

Développement et sécurité sont les deux faces d'une seule médaille, explique le cardinal sud-africain Desmond Tutu dans l'avant-propos du premier Human Security Report ${ }^{11}$. Le rapport du Programme des Nations unies pour le développement (PNUD) sur le développement humain de $2005^{12}$ se penche également sur les liens étroits entre «le développement, la sécurité et la démocratie». Sa réflexion repose sur l'observation que les investissements ne sauraient être durables s'ils peuvent à tout moment être détruits par la violence ou lorsque les

9 Caritas Suisse, Action pour le Carême, EPER, Swissaid, Ziele und Strategie für die gemeinsamen zivilgesellschaftlichen Friedensprogramme in Kolumbien, Mexiko, Guatemala, mars 2004.

10 Voir D. Senghaas, «Frieden als Zivilisierungsprojekt (Zivilisatorisches Hexagon)», dans Den Frieden denken, D. Senghaas (Hrsg.), Frankfurt a. Main, Suhrkamp Verlag, 1995.

11 Human Security Centre, Human Security Report 2005 : War and Peace in the 21st Century, New York; Oxford, Oxford University Press, 2005.

12 PNUD, Rapport mondial sur le développement humain 2005, Paris, Economica, 2005. 
gens qui vivent dans l'arbitraire et la peur des représailles ne peuvent se risquer à prendre des initiatives. La pauvreté et la répression sont les pires violations des droits humains fondamentaux. Les personnes qui doivent lutter jour après jour pour leur survie ne peuvent pas participer à la vie politique ni au débat démocratique. Selon le Rapport mondial sur le développement humain, 22 des 32 pays les plus pauvres ont été pris depuis 1990 dans des conflits armés. La prévention de la violence et la protection contre la violence sont donc également une priorité des ouvres suisses d'entraide en matière de développement et de promotion de la paix, que ce soit dans les régions formellement reconnues comme en crise ou dans les pays et les régions où la violence est élevée.

L'une des conditions nécessaires au processus de réconciliation durable est la mise sur pied d'un débat public aussi large que possible par des organisations non gouvernementales présentant un certain poids. C'est ce qui ressort de l'expérience des commissions pour la vérité et la réconciliation d'Afrique et d'Amérique latine, et que confirment plusieurs experts renommés ${ }^{13}$. Cette démarche paraît d'autant plus importante lorsqu'on sait que la moitié des pays sortant d'une guerre retombent dans un conflit armé dans les cinq ans suivant la signature d'un traité de paix.

\section{Regroupement des groupes}

de plaidoyer (advocacy) pour la politique de paix en Suisse

Alors qu'Alliance Sud se concentre sur les points cruciaux que sont, pour les politiques de sécurité et de paix, le commerce, la finance, l'environnement et la gouvernance mondiale, les autres forces engagées dans la politique de paix sont, en Suisse, moins bien regroupées. Plusieurs ONG travaillent dans différentes directions: certaines sur le front des «armes légères et exportations d'armes», d'autres pour une meilleure intégration des femmes dans les processus de politique de paix, pour le droit des minorités et des peuples indigènes, ou encore à la question de l'impunité. Pour les organisations dont la mission est intérieure, le plaidoyer pour la politique de paix se traduit aussi par un engagement pour les personnes qui, dans le pays, se trouvent dans une situation sociale précaire. Il existe des alliances de groupes de plaidoyer en politique des réfugiés et de l'asile analogues à ce qu'on trouve dans le développement.

Le travail de plaidoyer pour la paix (advocacy) est de nature plutôt concrète, entre autres parce que la réflexion sur la politique de paix se réfère beaucoup à l'équilibre des pouvoirs, aux besoins et aux opportunités, et parce que, en ce qui concerne les conflits globaux d'intérêts, l'influence des ONG est généralement insuffisante. La concentration sur un choix plus restreint de sujets, un lien entre le politique et le pratique pourraient redonner plus de vigueur au plaidoyer suisse pour la paix.

À côté de leur travail de développement dans le Sud, les œuvres d'entraide plaident pour des changements au Nord. Elles montent des campagnes d'information, organisent des événements culturels et lancent des débats sur la politique de développement et de paix ainsi que sur des questions socio-économiques.

13 Conférence internationale de la Division politique IV du DFAE et du Centre pour la promotion de la paix (KOFF) à Neuchâtel sur le thème «Transitional Justice», 23-24 octobre 2005. 
Différents projets innovateurs lancés ces vingt dernières années par les organisations de coopération et les œuvres d'entraide font aujourd'hui quasiment partie de la vie quotidienne, comme le commerce équitable et le marketing culturel en faveur de la littérature et du cinéma d'autres continents. Au sens large du terme, comme contribution à la compréhension entre gens et cultures et à des échanges commerciaux équilibrés, ces initiatives sont une promotion de la paix.

Par contre, le travail d'information et les actions sur certains conflits qui n'apparaissent pas tous les jours aux nouvelles ont de la peine à capter l'attention du public et sont financièrement moins soutenus que les projets concrets pouvant présenter des résultats visibles. Les organisations non gouvernementales d'autres pays européens renforcent leur travail de plaidoyer, par exemple en ouvrant leurs propres bureaux spécialisés ${ }^{14}$. On n'observe pas clairement ce type d'évolution en Suisse.

\section{Un travail de développement et de promotion de la paix tenant compte des conflits}

En soutenant le changement social par le bas, les organisations locales et les organisations internationales non gouvernementales se meuvent sur un terrain très délicat. Vouloir orienter les dynamiques conflictuelles sur la voie de la désescalade peut faire surgir bien des contradictions. C'est pourquoi la majorité des œuvres suisses d'entraide s'efforcent d'intégrer des mesures de sensibilisation aux mécanismes conflictuels dans leur gestion des programmes et des projets. Dans certains pays, comme par exemple en Colombie et au Népal, des formations et des évaluations ont été organisées selon l'approche do no harm en collaboration avec la Direction du développement et de la coopération (DDC). Il s'agit d'une part de vérifier les effets sur la paix ou les conflits des programmes d'aide d'urgence et de développement, cela dans toutes les régions, afin de s'assurer que le transfert de ressources ne favorise pas l'escalade de la violence. Questions centrales: Qui profite de l'aide? Qui en est exclu? Les options du projet renforcent-elles ou affaiblissent-elles les forces pacifiques locales?

Ce débat est jugé important par de nombreux collaborateurs de la coopération internationale mais ils estiment souvent insuffisante l'application de la méthode do no harm au seul niveau des projets. Il faudrait envisager l'ensemble de la situation et y inclure la responsabilité des donateurs multilatéraux et des organismes publics ou économiques, dont les stratégies contrecarrent trop souvent la promotion de la paix.

Enfin, le travail de développement et de promotion de la paix adapté aux réalités des conflits met forcément l'accent sur la bonne gouvernance. Dans les conflits intérieurs, la confiance entre la société civile et les instances étatiques est souvent compromise et la société civile est fréquemment très fragmentée. Les organisations non gouvernementales sont intéressées au renforcement de l'Etat, qui peut assurer de meilleures conditions-cadres, entre autres pour le respect des droits humains et pour la participation de la société civile, mais elles se doivent de maintenir une distance critique par rapport à l'Etat.

14 Un bon exemple en est l'oNG allemande Brot für die Welt. 
Les œuvres d'entraide peuvent avoir accès à la société civile au travers de leurs organisations partenaires et disposent ainsi de bons réseaux et de contacts importants permettant de construire des rapports de confiance avec les protagonistes du conflit. Cette orientation très fortement ciblée sur les partenaires exige toutefois des œuvres d'entraide de procéder à un examen critique de leur rôle dans le conflit, d'être capables d'analyser les situations avec suffisamment de distance et de vérifier l'efficacité des programmes de promotion de la paix. Dans la gestion des conflits et la promotion de la paix, il est indispensable de tenir compte des actions, des intérêts et des craintes de tous les acteurs et de rester ouvert aux contacts les plus divers ainsi qu'aux modes de règlement des conflits allant du constructif au plus inattendu, tout en restant inflexible sur les droits fondamentaux.

Les rapports entre la logique de la politique de paix et la logique de la politique des droits humains peuvent parfois être tendus. Il faut cependant toujours se rappeler que «dans la promotion de la paix - où les approches peuvent être très différentes et inattendues - nous devons nous orienter selon les droits humains fondamentaux et leur respect», comme le confirme un représentant du Département fédéral des affaires étrangères (DFAE) ${ }^{15}$. La durabilité d'un accord de paix dépend de son respect des droits humains. Inversement, en matière de droits humains, il s'agit d'agir de manière prudente, en tenant compte de tous les facteurs intervenant dans un conflit.

\section{Une approche fondée sur les droits humains}

La majorité des organisations de développement fondent leurs activités sur la défense des droits humains. Ces droits sont à la base de l'action non violente et du vivre-ensemble plus équitable, au niveau national comme sur le plan international, parce qu'ils formulent clairement les grandes questions politiques, culturelles et économiques. Ils bénéficient également - du moins sur le papier, dans les traités et dans les déclarations politiques - du soutien de la majorité des pays représentés aux Nations unies.

L'une des conditions préalables au bon fonctionnement des droits humains est que les gens connaissent leurs droits et que les populations de tous les pays s'efforcent d'en assurer et d'en élargir l'application ${ }^{16}$.

Felix Hafner, professeur de droit public à l'Université de Bâle, décrit ainsi les rapports entre le travail des organisations non gouvernementales et l'engagement pour les droits humains: «Le rapport entre l'activité des organisations sociales et la protection des droits humains est direct, même s'il n'est pas toujours perçu. Au niveau planétaire, les organisations internationales non gouvernementales se situent sans autre dans le cadre de la protection des droits humains. Au niveau national ou local cependant, le travail des organisations d'intérêt général n'est généralement pas jugé selon les critères des droits

15 Wolfgang Amadeus Bruelhart, «Mainstreaming of Human Rights », conférence, 28 mai 2004.

16 Voir G. Somer, «Menschenrechte und Friedenskultur», document de travail de la réunion "Globalisierung als Aufgabe - Handlungsmöglichkeiten und Gestaltungsoptionen der Politik», Evangelische Akademie Loccum, 1999. 
humains. On les considère plutôt comme des mutuelles d'assistance sociale. ${ }^{17}$ Pour Felix Hafner, le rapport avec les droits humains est double: «D'une part, il existe un besoin dans la société de travailler pour et avec les personnes défavorisées; d'autre part, cette tâche incombe à l'Etat, cette obligation étant ancrée dans de nombreuses constitutions. ${ }^{18}$ Que la tâche soit accomplie par l'Etat luimême ou par une organisation non gouvernementale est secondaire. Mais les organisations non gouvernementales qui, à la demande de l'Etat, prennent en charge une tâche publique deviennent, en matière de droits fondamentaux et de droits humains, l'interlocuteur des personnes qui leur sont confiées. Ceux qui prennent en charge des tâches publiques sont liés par les droits fondamentaux et sont obligés de contribuer à leur défense.

Les œuvres suisses d'entraide soutiennent les bureaux de consultation juridique, les associations de défense des droits humains, les missions d'observation du respect des droits humains ainsi que les groupes de pression internationaux et les réseaux d'organisations de défense des droits humains et de développement qui cherchent à influencer la politique des pays industrialisés et des pays en développement ainsi qu'à agir sur le plan multilatéral. Leur objectif est de protéger les populations contre la violence et l'arbitraire et de leur permettre d'exercer leurs droits politiques et civiques. Leur objectif est également, de plus en plus fréquemment, de réclamer l'application des droits économiques, sociaux et culturels auxquels les gouvernements ont souscrit par des conventions internationales, et de hâter la traduction des normes en lois nationales.

Outre leur travail d'explication et d'information pour la population, les organisations utilisent les médias pour rendre publics certains cas de violation des droits humains puis cherchent à les amener devant les tribunaux, par exemple lorsqu'il s'agit de prostitution enfantine, d'expropriations illégales ou de nonapplication de lois sur la réforme agraire.

Pour déposer plainte pour violation des droits humains, il est important de disposer de preuves, tout particulièrement lorsque les violations ont été perpétrées au cours de conflits armés étalés sur des années. La lutte contre l'impunité - par des procès exemplaires ou par le travail de commissions pour la vérité et la réconciliation - passe par un débat public sur les droits fondamentaux, le sens de la justice et la responsabilité. Dans les pays qui ont sombré dans la guerre ou la violence, en particulier ceux dont la société civile n'a pas traditionnellement une vie associative indépendante, le passage d'un régime de la violence à celui de la paix ne peut pas se faire de lui-même. Cette transformation est un long processus dépendant du soutien continu d'acteurs nationaux et internationaux.

Malgré la reconnaissance universelle des droits humains et leur inscription dans les législations nationales, la question du caractère contraignant des systèmes juridiques, de leur clarté et de leur acceptation reste ouverte. Alors que les donateurs multilatéraux et étatiques incluent rarement dans leurs plans les mécanismes traditionnels locaux de règlement des conflits, les œuvres d'entraide orientées vers le partenariat leur attachent une grande importance, souvent pour éviter d'être accusées d'impérialisme culturel. A quoi s'ajoute parfois cette

\footnotetext{
17 F. Hafner, «Menschenrechte und die Rolle der NGO, Aufgabenerfüllung für den Staat», Neue Zürcher Zeitung, 3. September 2001.

18 Ibid.
} 
opinion selon laquelle la liberté culturelle impose bon gré mal gré la défense des valeurs et coutumes traditionnelles. Le rapport du PNUD sur le développement humain de 2004 répond à cela que la liberté culturelle doit élargir les possibilités de choix des individus plutôt que de faire de la sauvegarde des valeurs et des coutumes une fin en soi. Le rapport plaide également pour une combinaison des systèmes juridiques, soit pour le pluralisme juridique. Le PNUD fournit quelques indications importantes pour le débat sur la réforme des droits coutumiers, en particulier des exemples d'adaptation du droit coutumier aux normes internationales qui n'ont fait qu'aggraver le sort de groupements qu'il s'agissait de protéger. C'est pourquoi il est conseillé de n'entreprendre de réforme d'un droit coutumier qu'en collaboration avec les intéressés ${ }^{19}$.

La dimension juridique, qui vise à assurer le respect des lois nationales et des accords internationaux ainsi que le développement des normes et des institutions juridiques, est un élément important du travail de défense des œuvres d'entraide. Le fait que cela ne suffit pas et que chaque société a besoin de relations économiques, politiques et sociales qui soient perçues comme étant justes, au-delà des frontières territoriales et culturelles, fondées sur la compréhension mutuelle, les contacts et le dialogue ainsi que sur les débats politiques et religieux, ici comme ailleurs - ce fait a été clairement illustré par les violentes manifestations soulevées par la «polémique des caricatures» au début de 2006.

\section{Défis et possibilités}

L'expérience acquise grâce aux programmes de paix communs, la recherche de stratégies adaptées et de coopérations possibles, les possibilités et les limites de la promotion - gouvernementale ou non - de la paix ont provoqué un vaste débat et un important processus d'apprentissage centrés principalement sur les questions suivantes.

- Quelles sont les conséquences du travail de développement et de l'aide d'urgence sur la dynamique des conflits et leur évolution vers la paix?

Les histoires et études de cas réunies par Mary B. Anderson et al. dans les ouvrages Do No Harm et Options for Aid ${ }^{20}$ montrent comment l'aide d'urgence peut aggraver les conflits. Il y a plusieurs décennies qu'une bonne partie des organisations en sont conscientes. Cependant, l'observation systématique et la prise en compte de cette question dans la planification, le suivi et l'évaluation des programmes et projets d'aide d'urgence et des programmes de développement sont un phénomène récent. De nombreuses œuvres suisses d'entraide ont entrepris un processus d'apprentissage et intégré ce type de réflexion dans leur culture interne ${ }^{21}$. Outre le processus international do no harm, cette évolution a reçu d'importantes impulsions de la Direction du

19 PNUD, rapport 2004, Neue Zürcher Zeitung, 28. Januar 2005, p. 9.

20 M.B. Anderson, Do No Harm, op. cit. M.B. Anderson (ed.), Options for Aid in Conflict: Lessons from Field Experience, Cambridge, Mass., The Collaborative for Development Action, 2000.

21 HEKS, Konzept Mainstreaming Friedensverträglichkeit in der Auslandarbeit, Mai 2005. Notice «Friedensverträglichkeit in der HEKS-Inland-Arbeit: Konfliktprüfung», Dezember 2004. Caritas, Umsetzungskonzept Konfliktsensibilität, 2005. 
développement et de la coopération (DDC) ${ }^{22}$. Par ailleurs, les œuvres d'entraide ont publié avec la Chaîne du bonheur une liste de contrôle des implications involontaires de l'aide d'urgence dans les conflits ${ }^{23}$. Les échanges d'expériences sont fréquents et les événements organisés par le Centre pour la promotion de la paix (KOFF) en sont également l'occasion, comme cela a été le cas après l'aide d'urgence aux victimes du tsunami en Indonésie et au Sri Lanka. Chaque organisation est responsable de ses projets et de ses programmes, lesquels doivent éviter d'accroître les tensions dans les zones de conflits. Cependant, leur engagement est remis en cause lorsque d'autres acteurs, en particulier de gros donateurs, ignorent ces précautions.

- Comment, dans les pays du Sud et de l'Est, les ouvres d'entraide peuventelles promouvoir les processus d'habilitation et le changement social pour les défavorisés tout en contribuant à la désescalade?

L'objectif final du processus d'habilitation (empowerment) est une société libre d'exclusion, de marginalisation, de discrimination et de violence. Les organisations qui soutiennent ce processus évoluent fréquemment dans un contexte conflictuel marqué par la violence, la répartition inéquitable des ressources et un climat d'incertitude. L'habilitation s'oppose à l'exclusion et à la violence et, dans ce sens, constitue une importante mesure de prévention. Cependant, la lutte pour l'accès aux ressources et pour la reconnaissance de ses droits peut entraîner une escalade de la violence. Ainsi, une organisation non gouvernementale qui s'engage de manière conséquente pour le droit à la terre et pour d'autres réformes en faveur des exclus se trouve embarquée dans un conflit et devient l'un des moteurs du changement.

- Comment les cuvres d'entraide peuvent-elles contribuer à la résolution non violente des conflits d'accès aux ressources et aux prestations?

Beaucoup d'organisations du Sud ont une longue expérience des stratégies et politiques non violentes. Il reste toutefois de la responsabilité des œuvres donatrices d'envisager lucidement les conséquences possibles de leur soutien, d'en discuter avec leurs partenaires et de suivre le processus de près. Cela nécessite des capacités personnelles et professionnelles qui ne sont pas données à tout le monde.

- Comment concilie-t-on la solidarité avec un groupe particulier et l'ouverture à toutes les parties, qui est l'une des exigences de la promotion de la paix?

La coopération au développement procède du sentiment de solidarité. Ce sentiment attache une valeur éthique au fait d'aider son prochain en difficulté. Dans leur communication, les œuvres d'entraide font fréquemment référence à des groupes tels que les pauvres, les spoliés, les femmes, les jeunes, les travailleurs, les paysans, les réfugiés et les victimes. Ce qui crée des liens particuliers avec tel ou tel groupe. Cependant, la promotion de la paix, en particulier la médiation dans les conflits et la recherche de moyens originaux de règlement des conflits, présuppose l'accès à toutes les personnes impliquées. S'il existe de bons exemples d'institutions et de

22 Swiss Development Cooperation (SDC), Conflict-Sensitive Program Management in the International Cooperation: Mainstreaming the Prevention of Violence (CSPM), October 2005.

23 Chaîne du bonheur, Liste de contrôle prévention et sensibilité à la thématique des conflits, mai 2005. 
personnes intègres et reconnues de divers côtés, par exemple dans les milieux ecclésiastiques (Mozambique, Soudan, Colombie), et qui ont clairement contribué à résoudre des conflits, on ne peut pas toujours parier sur un tel succès.

- La collaboration étroite et de longue durée avec des organisations partenaires est-elle une chose heureuse ou constitue-t-elle un risque pour la promotion de la paix?

Les partenariats de longue date témoignent d'un bon rapport de confiance et permettent, le cas échéant, un dialogue ouvert sur les questions difficiles. Mais l'inverse n'est pas exclu: les partenariats traditionnels sont parfois difficiles à remettre en cause, le respect des critères de travail n’y est parfois plus assuré avec la diligence souhaitable. Dans les situations de conflit, le positionnement des organisations partenaires change au fil des ans. On leur confie parfois de nouvelles tâches pour lesquelles elles n'ont ni les capacités ni les compétences. Le dialogue qui doit s'instaurer entre les collaborateurs d'œuvres d'entraide est un dialogue exigeant, entre la critique et le renforcement des liens de confiance.

- Peut-on concilier un travail conséquent de défense des droits humains avec une pensée orientée vers la politique de paix, laquelle recourt parfois à des solutions inattendues?

Cette tension se manifeste surtout lors de négociations de paix ou lors de prises de position politiques au niveau national comme, par exemple, en matière de droit au retour des réfugiés, de sanction des violations des droits humains, de libertés politiques et de droits participatifs. Il peut arriver que des groupements de la société civile empêchent la conclusion d'accords de paix en exigeant l'application de certains droits et la condamnation de certains responsables. Mais on constate aussi que, dans la pratique, les accords de paix qui mettent les questions de droits humains entre parenthèses ne tiennent généralement pas longtemps. Les œuvres d'entraide et, avant tout, leurs organisations partenaires du Sud et de l'Est doivent sans cesse se demander où et comment s'engager pour le respect et le développement des droits humains, tout en cherchant les solutions temporaires permettant d'endiguer la violence.

- Comment les organisations évitent-elles de surestimer leur rôle et d'éveiller des attentes contre-productives?

L'utilité de l'engagement pour la paix et la cohabitation pacifique est indiscutable. Toutefois, pour les œuvres d'entraide, la différence entre coopération au développement, promotion ciblée de la paix ou transformation des conflits est un sujet de discussions internes parfois difficiles. Parce que, finalement, toutes ces activités, quel qu'en soit le domaine, devraient conduire à la paix et à l'équité. Mary Anderson montre, dans Reflecting on Peace Practice ${ }^{24}$, que de nombreuses organisations poursuivent leur lutte en posant des exigences exagérées, ce qui les conduit à l'échec. Il faut être réaliste sur les limites et les possibilités des différents projets. L'amélioration des modes de

24 Collaborative Learning Projects, Reflecting on Peace Practice Project, Cambridge, Mass., Collaborative Learning Projects, 2004. 
culture ou la construction d'infrastructures - même réalisées de manière participative - contribuent sans doute, à long terme, à l'amélioration de la vie des habitants d'une région, mais ne contribuent pas forcément à la réduction à court terme de la violence ou à la résolution d'un conflit. Une simple mission d'observation ne permet pas d'obtenir la «grande paix» entre les Etats ou les régions. Une initiative de paix géographiquement limitée, une collaboration locale au développement adaptée aux réalités des conflits ou une aide d'urgence ponctuelle peuvent aider les habitants d'une région à vivre en paix et renforcer les mécanismes de règlement des conflits. Cependant, sans bases solides ni structures supérieures, il est difficile et peu probable de mettre fin aux violences et de créer des conditions de paix durables.

- Comment les programmes de coopération au développement (p. ex. pour la sécurité alimentaire ou la formation) s'articulent-ils avec la promotion de la paix afin d'obtenir un effet aussi durable que possible sur la paix?

La majorité des œuvres suisses d'entraide travaillent dans des régions touchées par des conflits, des crises, des guerres ou une violence généralisée. Par le passé, et pour les raisons mentionnées plus haut, elles se sont rarement demandé comment contribuer systématiquement à la réduction des violences ou à la résolution à court ou moyen terme des conflits (working around conflict, voir la contribution de Thania Paffenholz au présent ouvrage). Le regard plus aiguisé que l'on pose sur la dynamique des conflits et sur les processus de paix a modifié la perception qu'on en a, mais il est encore difficile, dans la pratique, de lier de manière directe les différents programmes et projets de coopération au développement ou d'autres préoccupations transversales comme les questions de genre, et la promotion ciblée de la paix. Cela parce que les œuvres d'entraide travaillent souvent dans les régions du pays non touchées par la violence (p. ex. dans le sud de l'Inde et non dans le nordest), parce que les organisations partenaires disposent de compétences précieuses pour la réalisation de leurs programmes et de leurs projets mais se trouveraient surchargées par l'ajout d'une composante promotion de la paix. En prenant des mesures adaptées aux réalités des conflits, les œuvres d'entraide essaient de chausser les «lunettes paix et conflits», c'est-à-dire de discerner en quoi leurs projets risquent d'accroître les tensions, mais aussi de mieux comprendre en quoi ils peuvent contribuer au règlement des différends. Quant à la surcharge, il est important pour l'éviter de pratiquer la mise en réseau avec d'autres acteurs spécialisés.

- Quelle position le travail de plaidoyer doit-il adopter face aux programmes opérationnels de développement?

Nous avons vu que l'engagement pour la paix à petite échelle, dans un contexte local limité, était important mais insuffisant. Il faut obtenir l'engagement des structures nationales et internationales œuvrant pour la paix, il faut des mesures et un contrôle politiques, il faut donc réclamer une bonne gouvernance. Les œuvres suisses d'entraide inaugurent et soutiennent à des degrés divers et à différents niveaux des stratégies de plaidoyer. Beaucoup d'entre elles soutiennent les organisations partenaires qui s'engagent pour les droits humains des personnes vivant dans des situations difficiles. Certaines sont également actives sur le plan politique intérieur, en Suisse, et parties prenantes de réseaux internationaux qui interviennent auprès des organisations 
internationales (ONU, UE, etc.). Dans la course aux ressources financières que se livrent les projets destinés au Sud et à l'Est, le travail thématique de plaidoyer et de lobbying risque d'être défavorisé. Il est souvent plus facile pour les communautés publiques comme pour les gros donateurs de financer des projets simples et facilement présentables plutôt que des mesures de plaidoyer moins visibles et dont l'utilité et la valeur ajoutée ne seront perceptibles que bien plus tard.

Le dénominateur commun aux questions ci-dessus, la réponse partielle dégagée par l'expérience et les évaluations, c'est l'importance des analyses systémiques, de la formulation claire des objectifs, du choix d'une stratégie adaptée, d'une gestion des programmes et des projets adaptée aux réalités des conflits, de la mise en réseau des programmes, et de la qualité du travail de communication. Autant de critères de qualité également applicables aux autres programmes de coopération au développement.

Il est également important que les donateurs et les organisations partenaires soient bien conscients de leur rôle dans la situation conflictuelle où ils interviennent et qu'ils perçoivent les opportunités mais aussi les limites de leur action. Il peut être plus efficace de se concentrer sur une compétence de base, comme le développement rural, et, par exemple, d'y soutenir l'intégration de mécanismes de règlement des conflits ou la mise en réseau d'acteurs de différents niveaux, que de lancer ses propres projets de paix insuffisamment ancrés dans la réalité.

Autres défis pour la promotion et la politique de la paix

Agenda mondial de la paix

Les discussions sur les dividendes de la paix au lendemain de la guerre froide se sont tues et l'on reste bien discret sur la ponction que les guerres et la violence imposent sur le budget des Etats et ceux du développement. A la fin 2005, les Etats-Unis avaient dépensé à eux seuls 251 milliards de dollars pour la guerre d'Irak, soit trois fois plus que le total du montant investi annuellement par l'ensemble des pays industrialisés dans la coopération au développement ${ }^{25}$. Ne sont pas inclus dans ce chiffre les dommages matériels et non matériels occasionnés en Irak et dans la région. "Il est scandaleux que les dépenses mondiales pour l'armement approchent à nouveau les plafonds de la guerre froide, avec les Etats-Unis représentant plus $45 \%$ de ces dépenses », déclare Friedensgutachten $2005^{26}$. Le Rapport mondial sur le développement humain du PNUD constate que nous vivons aujourd'hui dans un monde hypertrophié sur le plan militaire et sous-développé sur celui des stratégies de sécurité humaine ${ }^{27}$. Même si le travail multilatéral a permis d'obtenir quelques résultats - par exemple pour la réforme de l'ONU, l'institution d'une nouvelle Commission de consolidation de la paix avec son bureau de coordination, la réorganisation du Conseil des droits humains -, il faut maintenant créer un mouvement énergique pour un agenda

25 «Hohe Folgekosten durch Irak-Krieg», Tages-Anzeiger, 7. Februar 2006, p. 24.

26 U. Ratsch et al. (Hrsg.), Friedensgutachten 2005, Münster, LIT Verlag, 2005.

27 «Une stratégie militaire surdéveloppée et une stratégie sous-développée pour la sécurité humaine» (PNUD, Rapport mondial sur le développement humain 2005, op. cit., p. 12). 
mondial de la paix et une économie mondiale de la paix. Les réseaux suprarégionaux et transnationaux d'œuvres d'entraide, en particulier ceux des Eglises et d'autres communautés religieuses jouissant d'un fort rayonnement (citons comme exemple les positions de l'Assemblée générale du Conseil œcuménique, en 2005 au Brésil), ont un rôle important à jouer à cet égard.

\section{La volonté politique d'appliquer son expérience et son savoir}

On sait ce dont la politique globale de la paix et sa promotion efficace auraient besoin: de mécanismes internationaux de détection précoce et de désescalade, mais aussi du renforcement des bases d'une stabilité durable par la reconstruction civile et sociale. On a bien compris la réalité et la complexité des conflits et ce savoir devrait intervenir dans tous les domaines politiques, dans les mesures prises par les Etats, les acteurs internationaux et l'économie, dans l'adaptation du type d'aide et de son calendrier (aujourd'hui, on dispose de moyens d'intervention humanitaires souvent importants pendant et peu après les conflits armés, mais d'un soutien insuffisant à la reconstruction économique, politique et sociale).

En Suisse, on ne manque pas d'initiatives, d'études et de politiques pour la paix, mais leur application se heurte trop régulièrement à des difficultés. Exemple: une évaluation externe mandatée par la DDC elle-même a conclu en 2004 que les fondements conceptuels du domaine «droits humains et Etat de droit» ne présentaient qu'une pertinence limitée et n'exerçaient donc qu'une mince influence sur le programme pratique de travail de la DDC. Selon cette étude, 2,6\% seulement du budget de la DDC pour la coopération bilatérale au développement ont été consacrés au domaine «droits humains et Etat de droit» ${ }^{28}$. Ces observations ont provoqué un débat interne et la redéfinition des grandes lignes d'action dans ce domaine. Une étude sur la politique extérieure de la Suisse constate que l'intégration de préoccupations relatives aux droits humains dans la politique économique extérieure du pays avait, ces dernières années, donné lieu à un intense débat ${ }^{29}$. L'auteur témoigne que ce débat a donné de la visibilité aux organisations non gouvernementales, aux conflits d'objectifs et aux incohérences, tout en faisant avancer la discussion. «Les services d'Etat ont dû s'occuper un peu plus des questions des droits humains et des problèmes de cohérence et ont moins pu se réclamer de la séparation de l'économie et de la politique. Sur les questions délicates et controversées telles que la Garantie contre les risques à l'exportation, les intérêts de l'économie ont en règle générale continué de primer sur les droits humains. Cela tient en bonne part au fait que les préoccupations en matière de droits humains sont conceptuellement et juridiquement insuffisamment ancrées. En particulier, le point de vue des droits humains n'a, à ce jour, pas trouvé sa place dans la procédure de prise de décision pour le commerce extérieur. $»^{30}$

28 Jon A. Fanzun, docteur en sciences politiques, «Schweizerische Menschenrechtspolitik seit dem Zweiten Weltkrieg - ein Überblick», Jusletter, 7. Februar 2004.

29 Ibid.

30 Ibid. 


\section{Faire apparaître les incohérences}

Peter Niggli, directeur d'Alliance Sud, fait ce constat: «Comme tous les pays industrialisés, la Suisse poursuit une politique Nord-Sud contradictoire. Les objectifs que vise son APD [aide publique au développement] ne s'accordent pas avec sa politique de commerce extérieur ou ses relations financières mondiales. Notre gouvernement en est conscient et cherche à rendre la politique helvétique plus cohérente. Ces efforts sont louables, mais il faut être réaliste: la cohérence n'est pas pour demain. Il apparaît ainsi que le terme de cohérence désignera longtemps encore le champ clos où l'on débat des conflits d'objectifs et des intérêts contradictoires de la politique suisse à l'égard du Sud. ${ }^{31}$ La Loi sur des mesures de promotion civile de la paix et de renforcement des droits de l'homme de 2004 apporte une réponse pragmatique à la question de la cohérence. Le gouvernement suisse s'y engage à être transparent et à mettre à plat les conflits d'objectifs pouvant surgir dans les mesures adoptées par ses différents services.

C'est là que les œuvres d'entraide peuvent faire intervenir le plaidoyer, thématiser les contradictions et faire comprendre au public en quoi tel objectif politique du gouvernement est dommageable et comment entreprendre de le redresser.

\section{Ressources disponibles pour l'adaptation des stratégies et la mise en réseau}

Les nouvelles bases légales adoptées ces dernières années, les initiatives, les programmes et la refondation de plusieurs organisations montrent que les questions de promotion de la paix et de gestion des conflits ont beaucoup progressé. Le nombre des activités ne devrait pourtant pas tromper: pour l'instant, il ne s'agit - sur le plan national comme sur le plan international - que d'un timide début de gestion préventive des conflits et de consolidation à long terme de la paix dans les sociétés sortant d'un conflit armé. On n'a pas encore vraiment étudié la manière dont la coopération au développement dans la société civile peut conduire à une paix durable. Il est toutefois évident que la coopération au développement ne peut être qu'une des composantes d'un «plan de paix» et que, tant pour la prévention des crises que dans les cas de conflits armés puis dans les situations d'après-guerre, l'engagement de l'ensemble des acteurs internationaux, nationaux et locaux est indispensable

Il faut améliorer les réseaux de compétences pratiques des différents acteurs et augmenter les effectifs ainsi que les ressources matérielles et en temps, afin de faire avancer la gestion préventive des conflits, la réflexion sur les leçons tirées, les nouvelles connaissances et l'adaptation correspondante des mesures ou stratégies. Les acteurs de la coopération au développement, les organisations spécialisées de plaidoyer et les institutions d'étude de la paix ne se rapprochent qu'avec hésitation les uns des autres, en Suisse comme dans les pays programmes des œuvres d'entraide - pays dans lesquels de nombreuses institutions ont vu le jour et tout un savoir-faire s'est constitué ; cette réalité devrait être mieux intégrée à la stratégie et au travail opérationnel des organisations gouvernementales et non gouvernementales.

31 P. Niggli, «Coopération au développement: les organisations d'entraide devraient-elles quitter le terrain?», dans Annuaire suisse de politique de développement 2004. Les onG de développement: rôles et perspectives, Genève, IUED, pp. 140-141. 
Après les années 1990, où la politique de paix a beaucoup mobilisé en Suisse les ONG et le débat public (votations populaires sur la politique de défense, séminaires, positionnement sur la réorientation de la politique extérieure suisse dans le cadre légal national et dans l'environnement international, campagnes contre les mines et pour l'interdiction des exportations d'armes), le débat s'est déplacé sur les mesures opérationnelles concrètes de promotion de la paix et sur une gestion des programmes et des projets qui tienne compte des conflits. Les succès rapides, voire spectaculaires - comme celui des organisations d'Eglises dans le processus de paix au Mozambique - n'ont pas vraiment été au rendez-vous, si bien que les attentes sont maintenant plus mesurées. Il est bien vrai que les acteurs confessionnels jouent toujours un rôle important au plus haut niveau de négociation des processus de paix, comme par exemple en Colombie et au Soudan, mais, bien souvent, le travail de longue haleine est assuré de manière moins spectaculaire par une base peu visible avec ses réseaux locaux, régionaux, nationaux et internationaux. Après l'explosion d'espoir des années 1990, on a repris conscience du fait que les processus de paix traînent en longueur, qu'il faut toujours compter avec des rechutes et que la paix et le développement sont interdépendants, si bien qu'il faut travailler simultanément sur les deux plans.

Les premières évaluations de grands programmes pour la paix ont mis en évidence les champs de tension, les difficultés et les limites dont les œuvres suisses d'entraide devront, à l'avenir, aussi tenir compte. Elles ont également montré qu'il était possible d'obtenir de bons résultats et que tout un potentiel reste à explorer dans les régions directement touchées par la violence, mais également chez nous, dans le travail de plaidoyer. Parce que nous sommes encore bien loin d'un agenda mondial pour la paix. 